\title{
Flamo: Filtering Algorithms for Location Based Publish/Subscribe on Moving Objects
}

\author{
Anu Mary Thomas ${ }^{1}$, Laiji George ${ }^{2}$ \\ ${ }^{1,2}$ AWH Engineering College, Calicut University, Department of Computer science \& Engineering, Kuttikkatoor, Kozhikode, India
}

\begin{abstract}
Flamo: The growth of social networks and mobile telephony has facilitated the real time propagation of location based services. Instant location based publish/subscribe system are unavoidable for next generation location based models. The existing works in this area are designed for static publishers/ subscribers. To explore the nearby local events and to get instant replies a push model system is necessary where user registers their interests and the server publishes instant relevant answers. In FLAMO we introduces an efficient publish/subscribe method that can easily publish location aware results even for moving subscribers. This uses an $R$-tree based index by integrating textual descriptions into $R$-tree nodes is used to achieve results. This method can support both conjunctive queries and ranking queries and also works for both static and moving objects.
\end{abstract}

Keywords: Subscription, Filter, LBS, Rt-Tree, Tokens

\section{Introduction}

Location Based services (LBS) has got significant attention from both Industrial and academic communities' .Its widely accepted because they can provide users with location-aware experiences. Existing LS systems employ a pull model or user-initiated model where a user issues a query to a server which responds with location aware answers. For example, if a subscriber (mobile user) wants to know the local events such as shows or exhibitions nearby, she issues a query to an LBS system, which returns answers based on users location and keywords. Through this method there occurs a chance for the user to miss some of the interesting events nearby. This mainly occurs due to communication delay or because of issuing explicit queries. To overcome this and to provide users with immediate results, a push model or server-initiated model is necessary for next generation location based services.

In the push model, subscribers can first register their spatialtextual subscriptions, to capture their interests into the system, and publishers post spatial-textual messages. This automatically calls for a high-performance location-aware publish/subscribe system to deliver messages to relevant subscribers. The subscriptions registered by subscribers and messages published by publishers include both spatial information and textual descriptions. This model brings a new user experiences to mobile users, and can help users to retrieve information without continuously issuing a query. There are many real-world applications using location aware publish/subscribe services. For example if we register to receive advertisements or news regarding a particular brand, the system pushes relevant results to mobile users based on their locations and contents they are browsing (e.g., webpages).

A publish/subscribe system should support tens of millions of subscribers and deliver messages to relevant subscribers in milliseconds [1]. Since messages and subscriptions contain both location information and textual description, it is rather costly to deliver messages to relevant subscribers. One big challenge of the existing methods is to support moving subscribers. Since the location of the subscriber changes continuously, it's difficult for the system to push suitable messages. To address this problem Flamo is introduced. In this method supports continuously moving queries and we capture the subscriber's location at each location. This avoids the chance of missing any events.

\section{Related Works}

Recently there are several studies related to the location based systems and some of them are listed below. Keyword Search on spatial databases [2] is an efficient method to answer top-k spatial keyword queries. An indexing structure called IR2-tree (Information Retrieval R-tree) which combines an R-Tree with superimposed text signatures is introduced. This happens because the signature of an IR2tree node is composed from all the signatures of its children. The tree traversal is based on the Incremental Nearest Neighbor algorithm. P. G. Cong, C. S. Jensen, and D. Wun proposed a new indexing framework for location aware top-k text retrieval [3]. This framework integrates the inverted file for text retrieval and the R-tree for spatial proximity querying to obtain an Inverted file R-tree. Nowadays, modern LBS applications generate a new kind of spatio-textual data, regions-of-interest (ROIs), containing region-based spatial information and textual description, e.g., mobile user profiles with active regions and interest tags. To satisfy search requirements on ROIs, a method called spatio-textual similarity search [4] proposed by J. Fan, G. Li, L. Zhou, S. Chen, and J. Hu: Given a set of ROIs and a query ROI, to find the similar ROIs by considering spatial overlap and textual similarity. X. Cao, G. Cong, C. S. Jensen, and B.Ooi states that a typical query takes a location and a set of keywords as arguments and returns the single spatial web object that best matches these arguments [5]. Its observed that user needs may exist that are not easily satisfied by a single object, but where groups of objects may combine to meet the user needs. Put differently, the objects in a group collectively meet the user needs. 


\section{International Journal of Science and Research (IJSR) ISSN (Online):}

Index Copernicus Value (2016): 6.14 | Impact Factor (2015): 6.391

To improve the effectiveness of interesting event dissemination to moving users, Long Guo , Lu Chen , Dongxiang Zhang build an efficient location-aware publish/subscribe system, named Elaps [6]. The system is based on the push model; mobile users are instantly notified whenever there is a matching event near them. Compared to Elaps have two distinguished features. First, it allows users to specify their interests with Boolean expressions, which provides better expressiveness in shaping an interest than keyword subscription. Second, it continuously monitors users' locations and sends nearby notifications in real time. To the best of the knowledge, Elaps is the first locationaware pub/sub system that takes into account moving subscribers as well as dynamic subscriptions and events. The main disadvantage of this system is that it cannot filter unwanted messages. Elaps is based on the push model and notifies mobile users instantly whenever there is a matching event around their locations. Moreover, Elaps can effectively monitor the subscribers without missing any event matching, and incur low communication overhead.

The method [1] uses a token-based index structure (called Rttree).It integrates each R-tree node with a set of tokens selected from subscriptions. Using the Rt-tree, we develop a filter-and-verification framework to efficiently deliver a message. To reduce the number of tokens associated with Rttree nodes, we select some high-quality representative tokens from subscriptions and associate them with Rt-tree nodes. This technique not only reduces index sizes but also improves the performance. Experiments on large, real data sets show that our method achieves high performance. It makes the following contributions: (a) Introduced a new computing model the location-aware publish/subscribe problem. (b) Introduces a novel index structure, the Rt-tree, by integrating high-quality representative tokens selected from subscriptions. Our method can support both conjunctive queries and ranking queries. (c) Using our proposed indexes, we develop efficient filtering algorithms and effective pruning techniques to improve the performance. (d) We present how to support dynamic updates efficiently.

Filtering Algorithms: This method uses efficient filtering algorithms to remove unwanted messages. The subscriber will receive only messages based on their subscriptions. It proposes a filter and verification framework. In filter step it finds a finds a set of candidate nodes. In verification framework verify the subscriptions in the leaf nodes. This mainly uses a MBR filter and a Token filter

\section{Filtering Algorithms for Location Based Publish/Subscribe On Moving Objects}

In this section we propose a new method called Flamo: filtering algorithms for location based publish/subscribe on moving objects. This method is introduced to publish messages to both static as well as moving objects. Most of the methods support only static subscribers. The main disadvantage of this system is that the subscriber may miss some interesting messages of their subscriptions. Flamo continuously captures the location of the subscribers using
GPS and using Geocoding to record the location information of the subscribers.

In a location-aware publish/subscribe system, subscribers register subscriptions to capture their interests. A subscription $\mathrm{s}$ includes a textual subscription $\mathrm{s}$. $\mathrm{T}$ and spatial information (location information) $\mathrm{s}$. $\mathrm{R}$, denoted by $\mathrm{s}=(\mathrm{T}, \mathrm{R})$. The spatial information is used to capture a subscribers most interested region and to push relevant messages. Let $S=\left(s_{1}\right.$, $\mathrm{s}_{2}, \ldots . \mathrm{s}_{\mathrm{i}}$ ) denote the set of subscriptions. Given a subscription $\mathrm{s}_{\mathrm{i}} \in \mathrm{S}$ and a message $\mathrm{m}$, a location aware publish/subscribe system delivers $m$ to $s_{i}\left(s_{i}\right.$ is called an answer of $m$ ), if the spatial constraint as well as textual constraint of the subscriptions overlap with that of the messages.

\subsection{Rt-Tree}

This method uses a token-based R-tree, called Rt-tree, by integrating tokens of subscriptions into R-tree nodes. Each subscription along with the subscriber's location creates the nodes of this Rt-tree. Rt-tree is a balanced search tree. Each leaf node contains between $\mathrm{b}$ and $\mathrm{B}$ data entries, where each entry is a subscription. The basic Rt-tree has no textual pruning power, hence high quality representative tokens selected and use these representative-token sets to replace Token Sets of $\mathrm{R}$ tree. Thus generates a Rt+-tree. From Rttree we want to prune unnecessary nodes and only visit a small number of pivotal nodes, where a node is a pivotal node if there exist answers in its leaf descendants. Thus when traversing the Rt+-tree, we only need to visit the pivotal nodes. When a message arrives in the system it will traverse the subscription tree and find the suitable subscriptions. Then these messages are pushed to the subscriber. However an Rt+-tree node may have large numbers of leaf descendants and it is expensive to check whether a node is a pivotal node. Based on this observation, proposes a filter. In the filter step the system continuously checks the location of subscriber and matches it with those of messages. If it is not matched, then messages will be discarded.

The FLAMO method supports both the static as well as moving subscribers. Hence for continuously checking the location of subscribers it uses a filter called Location based filter. This will automatically update the location details of the subscribers and will rearrange the tree nodes. When a message is posted by a publisher, a radius area is also set. This is to ensure that the messages are send to the subscribers within that range. If a subscriber is present in the message area of publisher then the message checks the Rt+- tree to identify whether that publisher is subscribed or not. Suppose a subscription on leaf node 1 is an answer of message $\mathrm{m}$. As $\mathrm{s}$ is an answer of $\mathrm{m}$, it will appear on l's ancestor nodes from the root to node 1 . Then the messages are checked using a similarity checker to make that message unique in the system. If all the above conditions are satisfied then the Message fetching module push-up the notifications to relevant subscribers. The system architecture of this method is given below 


\section{International Journal of Science and Research (IJSR) \\ ISSN (Online):}

Index Copernicus Value (2016): 6.14 | Impact Factor (2015): 6.391

\subsection{System Architecture}

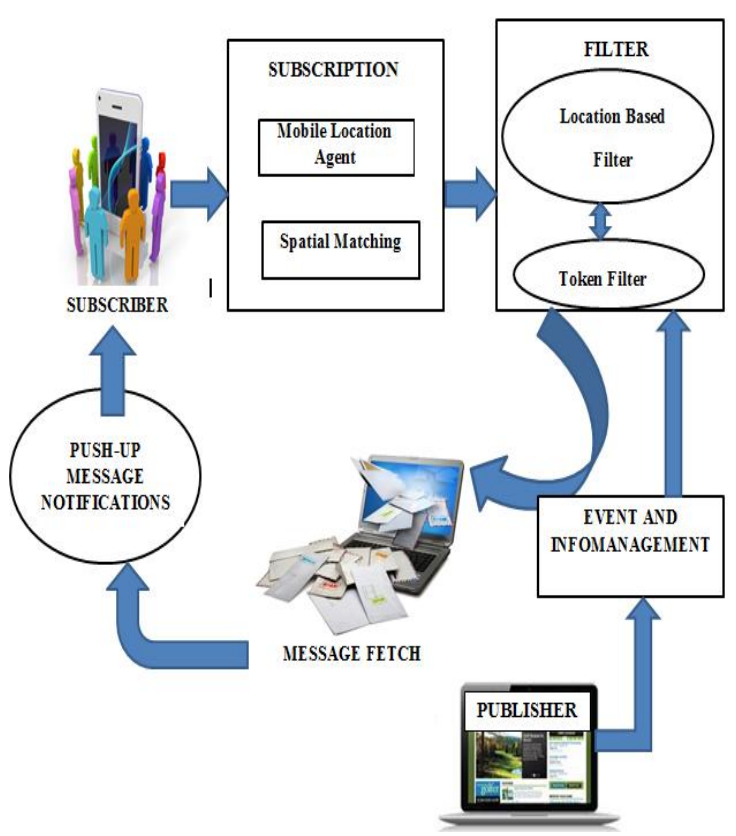

Figure 1: FLAMO Architecture

Subscriber: It is designed as a mobile user in this system. When the subscriber enters into the system they register their subscriptions such as area of interest as preferences. When suitable subscribed messages arrive it will be pushed to the subscriber.

Publisher: Here the publisher is the one who posts messages into the system. They post their latest notification into the system and the system delivers these messages to the suitable subscribers. Here the publishers are static.

Filter: There are two types of filter used in this system. 1) Location based Filter: They filter the messages according to the location of subscriber.2)Token filter: They filters the messages by checking the contents of subscriptions as well as message contents.

\section{Methods}

\subsection{Steps of Location based Filtering}

Input: Message list, Location Information

Output: Messages satisfying the location.

1) Update the location information of subscriptions.

2) Then check whether the subscriber is within the radius limit of message.

3) If yes then messages are filtered.

4) Else messages are discarded

\subsection{Location Update Steps}

1)For each subscriber update a set $S=s 1$, s2, sn for each location.

2)Re-arrange Rt-Tree leaf nodes by performing Rt+-tree algorithm.

3)For each location of subscriber the algorithm will rearranges the tree nodes.

\subsection{Message Search Algorithm}

Input: A subscriber set $\mathrm{S}$, message set $\mathrm{m}$.

Output: messages to the subscriber.

1. Push messages to each subscriber in S.

2. Search (location, place, Subscriptions)into a set $P$

3 . Then check whether the subscriber is within the radius limit of message.

4. Check whether the message fall into the preference set $P$.

5 . If condition is satisfied messages are posted.

6. Else messages are discarded

\section{Conclusion}

In this paper we studied about location aware publish or subscriber on moving objects. The existing method works on static subscribers. Hence here we introduced a new filter called location based filter.The system continuously captures the location of subscribers and then efficient messages are pushed. To reduce the size of Rt-tree a tree structure called Rt+-tree is used. This improves the performance of this system.

\section{Future Scope}

With the rapid progress of mobile Internet and the growing popularity of smartphones, location-aware publish/ subscribe systems have recently attracted significant attention. It is possible to establish a future extension to the current work. In current system the messages are delivered to the relevant subscribers without any delay. Since subscribers have different preferences some subscribers prefer messages with high spatial proximity and some subscribers pay more attention to messages with high textual relevancy.So this learning can be including as a future extension in to the existing system.

\section{References}

[1] Minghe Yu, Guoliang Li, Member, IEEE, Ting Wang, Jianhua Feng, and Zhiguo Gong, "Efficient Filtering Algorithms for Location-Aware Publish/Subscribe ": IEEE transactions on knowledge and data engineering, VOL. 27, NO. 4, APRIL 2015.

[2] I. D. Felipe, V. Hristidis, and N. Rishe, "Keyword search on spatial databases": in Proc. Int. Conf. Data Eng., 2008, pp. 656665.

[3] P. G. Cong, C. S. Jensen, and D. Wu, "Efficient retrieval of the top-k most relevant spatial web objects": Proc. VLDB, vol. 2, no. 1, pp. 337348, 2009

[4] J. Fan, G. Li, L. Zhou, S. Chen, and J. Hu, "Seal: Spatiotextual similarity search": Proc. VLDB Endowment, vol. 5, no. 9, pp. 824835, 2012

[5] X. Cao, G. Cong, C. S. Jensen, and B.Ooi, "Collective spatial keyword querying "in Proc. ACM SIGMOD Int. Conf. Manage. Data, 2011, pp. 373384.

[6] Long Guo , Lu Chen , Dongxiang Zhang, Guoliang Li y4, Kian-Lee Tan , Zhifeng Bao, "Elaps: An Efficient Location-Aware Pub/Sub System": , NExT Search Centre (grant R-252-300- 001-490),2015

\section{Volume 5 Issue 6, June 2016 www.ijsr.net}




\section{Author Profile}

Anu Mary Thomas received the B Tech degree in Computer Science and Engineering from university of Calicut in 2014 and she is currently persuading her M-tech in Computer Science and Engineering from Calicut University.

Laiji George received the B Tech and M Tech degrees in Computer Science and Engineering from Cochin University and Anna University in 2011 and 2014, respectively. She is working with AWH Engineering College since December 2011.

Volume 5 Issue 6, June 2016 www.ijsr.net 